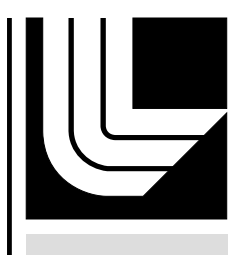

LAW RENCE LIVERMORE N A TIO N A L LABORATORY

2005 DOE Computer Graphics Forum Site Survey

Springmeyer, Rebecca, Brugger, Eric

April 25, 2005 
This document was prepared as an account of work sponsored by an agency of the United States Government. Neither the United States Government nor the University of California nor any of their employees, makes any warranty, express or implied, or assumes any legal liability or responsibility for the accuracy, completeness, or usefulness of any information, apparatus, product, or process disclosed, or represents that its use would not infringe privately owned rights. Reference herein to any specific commercial product, process, or service by trade name, trademark, manufacturer, or otherwise, does not necessarily constitute or imply its endorsement, recommendation, or favoring by the United States Government or the University of California. The views and opinions of authors expressed herein do not necessarily state or reflect those of the United States Government or the University of California, and shall not be used for advertising or product endorsement purposes.

This work was performed under the auspices of the U.S. Department of Energy by University of California, Lawrence Livermore National Laboratory under Contract W-7405-Eng-48. 


\title{
2005 DOE Computer Graphics Forum Site Survey
}

\author{
Lawrence Livermore National Laboratory
}

\section{Site Information}

Site Name

Site Division or Group

Site Representative

Site Rep Contact Data
Lawrence Livermore National Laboratory

Computation groups: IMG, Data, CASC

Eric Brugger and Becky Springmeyer

P.O. Box 808, L-557, Livermore, CA 94550

Eric (925) 423-1293, brugger1@llnl.gov

Becky (925) 423-0794, springme@1lnl.gov

Fax: (925) 422-3389

\section{What Does Your Group Do?}

Define the Ongoing Mission of your Group

The Information Management and Graphics Group supports and develops tools that enhance our ability to access, display, and understand large, complex data sets. Activities include developing visualization software for terascale data exploration; running two video production labs; supporting graphics libraries and tools for end users; maintaining four PowerWalls and assorted other advanced displays; and providing integrated tools for searching, organizing, and browsing scientific data.

The Data group supports Defense and Nuclear technologies (D\&NT) Directorate. The group's visualization team has developed and maintains two visualization tools: MeshTV and VisIt. These are interactive graphical analysis tools for visualizing and analyzing data on two- and three-dimensional meshes. They also provide movie production support.

Researchers in the Center for Applied Scientific Computing (CASC) work on various projects including the development of visualization and data mining techniques for terascale data exploration that are funded by ASC. The researchers also have LDRD projects and collaborations with other lab researchers, academia, and industry.

Describe Activities over the Previous Year

This year we expanded our production Linux visualization cluster operation to include three clusters, with two additional small machines currently being procured. We have two SGI vis servers as well. The IMG group has just moved into the new Terascale Simulation Facility, which includes a classified theater (still under construction), an unclassified vis theater, a vis developer's lab, a vis computer floor and deployment workshop, and video production labs. This year we continued to provide the traditional graphics group consulting and video production support. We maintained three PowerWalls and a host of other displays. 
The ASC projects have delivered new versions of visualization and scientific data management tools to end users and continue to refine them. VisIt had eight releases during the past year, ending with 1.4.2. We released version 1.2 of Hopper, a Java application for managing and transferring files, and version 5.0 of SimTracker, a calculation summary tool. The MIDAS remote viz system was released as 0.8 .8 this year. We continue to develop Blockbuster and Telepath, as well as tools to apply the research from the ViSUS project and a project for visualizing large molecular dynamics calculations. Information on these tools and efforts can be found on our DVS web pages http:// www.llnl.gov/icc/sdd/img/infrastructures.shtml. Specific references appear below).

Describe Activities Planned for the Upcoming Year

Plans include deploying additional production Linux clusters, including one to drive the classified theater in the TeraScale Simulation Facility, and a 256-processor machine to support visualization of BlueGene/L data. We will release new versions of the various inhouse tools we support. Priorities include applying our visualization tools and techniques to the large data sets that will be generated on BGL and the machines associated with the Purple contract.

Research into data mining and visualization techniques continues with an increasing focus on data comparisons and richness of information expression. The group will host a number of summer students working on a range of topics. We will continue to provide consulting and support services in graphics and video production.

\section{Whence Funding?}

The IMG Group is funded by ASC as well as other "core" and "institutional" sources.

CASC is funded by LDRD, ASC, and outside sponsors.

The Data \& Vis group is funded by ASC and other "core" DNT funds.

\section{Current Resources}

\section{Current Hardware}

Compute servers: See http://www.llnl.gov/icc/resources.html for details on the compute servers and storage systems.

Vis servers: We have two production servers on the unclassified side and three servers in our secure computing facility.

pvc Linux production cluster: 64 nodes (Dual 2.4Ghz P4 2GB RAM), Quadrics, nVidia GeForce4, nVidia FX3000G, shared lustre disk.

gviz Linux production cluster:

64 nodes (Dual 2.8Ghz P4 2GB RAM), Quadrics, nVidia 5950 Ultra, $\sim 3$ TB disk via lustre.

sphere Linux production cluster: 
96 nodes (Dual 2.8Ghz P4 2GB RAM), Quadrics, nVidia NV6800GT, shared lustre disk

SGI Onyx-2: 40 R12K 300 MHz CPUs, 17.6 GB RAM, 10xIR, 10 TB

disk.

SGI Onyx 3800: 96xR12K, 400 MHx CPUs, 96GB RAM, 4xIR, 5 TB

disk.

Video production: Our two labs include Octanes, PCs, Accom DDR, desktop video editors, and assorted recorders, monitors, mixers, and software packages.

Vis developer's lab (to be renewed in the TSF): Assorted small systems and a fourprojector powerwall driven by multiple Linux clusters.

D\&NT: B Program has migrated away from SGI systems and X Terminals to Linux systems with 100 Mbit -> Gigabit ethernet and nVidia or ATI graphics cards for the desktop. A Program is continuing to explore Linux systems with Gigabit ethernet and nVidia graphics cards.

Current Software

AVS, EnSight, IDL, VisIt, MeshTV, NCAR, assorted other vis tools, utilities and translators, xmovie, Blockbuster, MIDAS, Telepath, ViSUS, and the TeraScale Browser. SDM sofware includes SimTracker and Hopper.

See http://www.llnl.gov/icc/sdd/img/ for a more complete overview.

ASC Vis: http://www.1lnl.gov/icc/sdd/img/viz.shtml

ASC SDM: http://www.llnl.gov/icc/sdd/img/scientific.shtml

VisIt: http://www.llnl.gov/visit

ftp://ftp.llnl.gov/pub/visit

Blockbuster: http://blockbuster.sourceforge.net/

MIDAS: http://www.llnl.gov/icc/sdd/img/midas.shtml

Hopper: http://www.llnl.gov/hopper/

\section{Current Staff}

In Livermore Computing and CASC:

About 18-19 FTEs funded by ASC

$\sim 9$ Visualization Research and Development team

6.2 Scientific Data Management team R\&D team

3.0 Deployment team

1.5 Graphics support and development team

3.0 Video production team

In the DATA and Vis group there are 6 people supporting the D\&NT Directorate.

\section{Planned (or Wished For) Growth}


Hardware Acquisitions

Additional Linux clusters and replacement PowerWall projectors. We will buy a vis cluster to support BGL and two small clusters to drive walls and for R\&D purposes.

Software Acquisitions

Additional licenses for commercial software as needed.

Personnel Acquisitions

No openings.

\section{Additional Comments}

